\title{
Analyzing the role of practice-assisting manpower and its impact in emergency medical care settings.
}

\author{
Won Joon Jeong ${ }^{1}$, Jun Ho Kang ${ }^{2}$, Won Suk Lee ${ }^{3}$, Hyunjin Kim ${ }^{4}$, Jihyon Kim', Janice Oh${ }^{5}$, Tae Sik \\ Hwang $^{6}$, O Yu Kwon ${ }^{7}$, Seung Whan Kim ${ }^{*}$ \\ ${ }^{1}$ Department of Emergency Medicine, Chungnam National University Hospital, Daejeon, Republic of Korea \\ ${ }^{2}$ Department of Emergency Medicine, Chungbuk National University Hospital, Cheongju, Chungbuk, Republic of \\ Korea \\ ${ }^{3}$ Department of Emergency Medicine, Eulgi University Hospital, Daejeon, Republic of Korea \\ ${ }^{4}$ Department of Emergency Medicine, Konyang University Hospital, Daejeon, Republic of Korea \\ ${ }^{5}$ Tulane School of Medicine, New Orleans, LA, USA \\ ${ }^{6}$ Department of Emergency Medicine, Haeundae Paik Hospital, Inje University, Pusan, Republic of Korea \\ ${ }^{7}$ Department of Anatomy, College of Medicine, Chungnam National University, Daejeon, Republic of Korea \\ ${ }^{8}$ Department of Emergency Medicine, College of Medicine, Chungnam National University, Daejeon, Republic of \\ Korea
}

\begin{abstract}
Purpose: To investigate and analyse the role of practice-assisting manpower in the emergency care centers of teaching hospitals for more efficient allocation of the workload in hospitals to provide better medical care.

Methods: We enrolled a total of eight practice-assisting manpower in four emergency care centers of teaching hospitals located in Daejeon Metropolitan City and Chungcheong Province areas. The current demographics and conditions of practice-assisting manpower and their work schedules were surveyed and collected. Board-certified emergency physicians from each hospital observed tasks performed by practice-assisting manpower for 60 minutes during both day and night shifts, and data collected were analysed by time-motion study method.

Results: The practice-assisting manpower participants were of certified first-ranked Emergency Medical Technician (EMT). The mean working hours per week were $42.5 \pm 3.3 \mathrm{~h}$. The total number of performed tasks during the study period was 275 items (day shift: 141, night shift: 134) in total observation time. Tasks performed were divided into three categories: procedure-assisting $(87$ items), treatment-assisting (169 items), and personal activities (19 items). The time consumed for procedure-assisting tasks, treatment-assisting tasks, and personal activities were recorded as $8 \mathrm{~h} 35 \mathrm{~min} 47 \mathrm{~s}, 4 \mathrm{~h} 27 \mathrm{~min} 41 \mathrm{~s}$, and 2 h $56 \mathrm{~min} 32 \mathrm{~s}$, relatively.

Conclusion: Though some items in both procedure-assisting and treatment-assisting tasks were classified as beyond the scope of work of EMT, it is observed that the role of practice-assisting manpower in emergency care is critical and significant for efficient patient care in the emergency care settings. This further suggests reconsidering the functions of practice-assisting manpower and reallocates duties among the workforce of emergency medical service providers for the present and future.
\end{abstract}

Keywords: Emergency care, Manpower, Emergency medical technicians.

Accepted on March 13, 2018

\section{Introduction}

Although the number of patients admitted to emergency facilities has been increasing steadily, the manpower needed to accommodate these patients has not increased accordingly.
Thus, the amount of work given to interns as well as doctors have become more rigorous and excessive than originally required, and without any ultimate solution to this problem, the probability of their overloaded work leading to medical 
malpractice may be high. In the process of revising medical school curriculum, the closing of internship-training programs has been a possibility [1] from which emergency facilities can expect to face chaos as a consequence. Therefore, it is to reallocate the amount of work in emergency facilities from overburdened professionals to practice-assisting manpower is more significant and necessary than ever.

Many hospitals have hired practice-assisting manpower including Emergency Medical Technicians (EMT) and nurses to work in the emergency care settings, but the type of work of these professionals have not yet obtained and recorded statistically in a national-level database completely. Thus we hope to investigate whether the hired practice-assisting manpower provides adequate assistance to the medical doctors with clinical duties. Furthermore, in the cases of terminating internship-training programs and thus shortage of internshiptraining manpower in the emergency care settings, we questioned the level of effectiveness of practice assisting manpower if substituted in clinical settings.

This study is based in university hospitals in the city near Daejeon Metropolitan City and Chungcheong Province regions of South Korea and uses time motion study method to investigate the current demographics and performance of the workforce, as well as the various types of tasks performed during work hours. In the future, this research can be used to estimate the number of practice-assisting professionals needed to maximize efficiency in an emergency care setting.

\section{Methods}

\section{Participants}

The subjects of the study were collected from four university hospitals in the city near Daejeon Metropolitan City and Chungcheong Province regions that currently use practiceassisting manpower in clinical care settings. They were contacted with the purpose of the study and asked to participate in the investigation. Two practice-assisting professionals per hospital were randomly selected. And they closely monitored for the study.

\section{Data collection}

The current demographics and conditions of the workforce, work schedules, and participants' personal information were gathered from the emergency department of individual hospitals. Each trial of monitoring consisted of $60 \mathrm{~min}$, and the selected observer for each hospital was a professional doctor who knows well of the emergency room protocol [2]. It was assumed that the subjects of the study were from either the second or third shift, and the monitoring hours were divided into day time (8:00-18:00) and night time (18:00-08:00). Observations were made twice a day, one hour per shift. The selected observers monitored the subjects in the person with stopwatches to record total performance time (seconds) taken for each task or used video recordings of the subjects performing tasks in a given time frame for analysis.
The duties of practice-assisting manpower were divided into procedure-assisting, treatment-assisting, and personal activities. Procedure-assisting tasks consisted of venous line insertion and blood samples, electrocardiography, splint application, wound dressing (wound cleaning, assistant roles during suture procedures included), other procedures (arterial blood gas analysis, chest compression in cardiopulmonary resuscitation, nasogastric tube insertion, etc. In addition, treatment-assisting tasks included providing basic information to patients (i.e. medical procedures conducted in the emergency rooms, discussions with healthcare providers), inputting data (i.e. patient confirmation, blood test results, electrocardiogram readings), hand washing and relocating during work hours. Lastly, personal activities included rest, personal study, use of technological devices such as smartphones, use of the restroom, and conversations which are not related to medical care.

\section{Data analysis}

The three divisions of duties were individually classified as one unit, and the total time ( $\mathrm{min}$ ) spent for each division were compared to total monitored hours to calculate percentages. The total monitored hours per week were $60 \mathrm{~min}$ of dayshift and $60 \mathrm{~min}$ of nightshift per subject. The data collected were statistically analysed in averages and standard deviations (significance level of 0.05). The statistical analysis package of SPSS 15.0 for Windows (SPSS Inc., Chicago, USA) was used in this study.

\section{Results}

The data were collected from four participating university hospitals in Daejeon Metropolitan City and Chungcheong Province regions of South Korea starting from September to November in 2013 for three months. In 2012, the average number of patients admitted to emergency care centers (ECC) was $46658 \pm 8188$. There was an average of $5.3 \pm 1.9$ persons of first-grade practice-assisting manpower in each hospital; five females and three males were randomly chosen for the study. Their working hours consisted of the following: one hospital had second shift system (twelve working hours per duty) and the other three hospitals had third shift system (eight working hours per duty). The practice-assisting manpower worked $42.5 \pm 3.3 \mathrm{~h}$ per week in average.

The data of practice-assisting manpower for each hospital are shown in Table 1. Two participants from each of the four hospitals were individually monitored for two hours, one during day and one during night, for a total of $16 \mathrm{~h}$. There were 275 total tasks observed, 141 from day shift and 134 from night shift, which were categorized into 87 procedure-assisting tasks ( 8 h 35 min $47 \mathrm{~s}$ ), 169 treatment-assisting tasks (4 h 27 $\min 41 \mathrm{~s}$ ), and 19 personal activities ( $2 \mathrm{~h} 56 \mathrm{~min} 32 \mathrm{~s}$ ) in Table 2.

Within the specific time spent on procedure-assisting tasks, intravenous line insertion with blood sampling and wound dressing were the most time-consuming tasks in total. There 
were 31 cases of blood sampling, highest in number of cases within procedure-assisting tasks and second highest in time consumption among all tasks. Wound dressing was observed to consume the most in total time as well as per treatment. The second most time consuming within procedure-assisting tasks was electrocardiography. It was also observed that other various activities that were not officially regulated for practiceassisting manpower were being performed, such as arterial blood gas analysis, nasogastric tube insertions, and digital rectal examinations. Within treatment-assisting tasks, patient confirmation and data input into the computer had largest number of tasks with most consumed time.

Lastly, in terms of procedure-assisting tasks, providing basic information, relocation, and hand washing were monitored respectively. As for personal activities, the practice-assisting manpower spent on average of $557 \mathrm{~s}$ per hour $(9 \min 17 \mathrm{~s}$ per hour).

Table 1. Current state of practice assistant and emergency manpower in the investigation.

\begin{tabular}{|c|c|c|c|c|c|c|c|c|}
\hline & $\begin{array}{l}\text { Annual } \quad{ }^{*} E D \\
\text { patients (persons) }\end{array}$ & $\begin{array}{l}\text { ED physician } \\
\text { (persons) }\end{array}$ & $\begin{array}{l}\text { ED } \\
\text { (persons) }\end{array}$ & resident & Intern (persons) & Nurse (persons) & $\begin{array}{l}\text { Practice } \\
\text { assistant } \\
\text { (persons) }\end{array}$ & $\begin{array}{l}\text { Average work hour } \\
\text { per week (h) }\end{array}$ \\
\hline A hospital & 40,081 & 9 & 12 & & 6 & 43 & 4 & 43 \\
\hline B hospital & 40,843 & 6 & 4 & & 6 & 34 & 5 & 40 \\
\hline C hospital & 57,718 & 5 & 1 & & 4 & 36 & 8 & 40 \\
\hline D hospital & 47,993 & 4 & 3 & & 4 & 29 & 4 & 47 \\
\hline
\end{tabular}

Table 2. Practice assistant's time spent according to procedural tasks in the emergency department.

\begin{tabular}{|c|c|c|c|c|c|c|c|}
\hline \multirow[t]{2}{*}{ Activity } & \multicolumn{3}{|c|}{ Time (h:min:s) } & \multicolumn{3}{|c|}{ Number of cases } & \multirow{2}{*}{$\begin{array}{l}\text { Average time per case } \\
\text { (s) }\end{array}$} \\
\hline & Day & Night & Total & Day & Night & Total & \\
\hline Procedure assistant & $03: 47: 38$ & 04:48:09 & 08:35:47 (53.7\%) & 40 & 46 & 86 & $359.84 \pm 373.5$ \\
\hline Blood sample, IV line & $01: 16: 55$ & $01: 05: 13$ & 02:22:08 (14.8\%) & 16 & 15 & 31 & $275.1 \pm 186.4$ \\
\hline Electrocardiography & $00: 34: 44$ & $00: 37: 52$ & $01: 12: 36(7.5 \%)$ & 11 & 10 & 21 & $207.43 \pm 159.3$ \\
\hline Splint apply & $00: 13: 52$ & $00: 16: 18$ & $00: 30: 10(3.2 \%)$ & 2 & 2 & 4 & $452.5 \pm 162.8$ \\
\hline Wound dressing & $01: 33: 17$ & 01:10:01 & 02:43:18 (17\%) & 7 & 8 & 15 & $653.2 \pm 605.3$ \\
\hline *Other procedures & $00: 08: 50$ & $01: 38: 45$ & $01: 47: 35(11.2 \%)$ & 4 & 11 & 15 & $430.3 \pm 462.3$ \\
\hline Treatment assistant & $02: 35: 58$ & $01: 51: 43$ & $04: 27: 41(27.9 \%)$ & 88 & 83 & 171 & $94.1 \pm 112.6$ \\
\hline Giving explanation & $00: 39: 10$ & $00: 11: 10$ & $00: 50: 20(5.2 \%)$ & 13 & 7 & 20 & $151 \pm 151.4$ \\
\hline${ }^{* *}$ EMR recording & 01:18:24 & $00: 55: 49$ & $02: 14: 13(14 \%)$ & 29 & 23 & 52 & $154.86 \pm 151.7$ \\
\hline Moving & $00: 20: 47$ & $00: 33: 03$ & $00: 53: 20(5.6 \%)$ & 24 & 36 & 60 & $53.8 \pm 36.7$ \\
\hline Hand washing & $00: 17: 37$ & $00: 12: 11$ & $00: 29: 48(3.1 \%)$ & 22 & 17 & 39 & $45.8 \pm 24.3$ \\
\hline Private activities & $01: 36: 24$ & 01:20:08 & 02:56:32 (18.4\%) & 13 & 6 & 19 & $557.47 \pm 634.9$ \\
\hline Total & 08:00:00 & 08:00:00 & $16: 00: 00(100 \%)$ & 141 & 135 & 276 & $208.8 \pm 317.9$ \\
\hline
\end{tabular}

*Other procedures: arterial blood gas analysis, chest compression in cardiopulmonary resuscitation, nasogastric tube insertion, digital rectal exam, gastric lavage, oral swab; ${ }^{* *}$ EMR: Electronic Medical Record.

\section{Discussion}

As previously mentioned, official national-level data regarding practice-assisting manpower have not been performed in Korea yet. This is due to many hospitals hiring workers without specific standards of tasks required of practice-assisting manpower, their scope of work, organizational status etc. In 2011, report [3] indicated that practice-assisting manpower has increased rapidly, especially in high-ranking general hospitals and smaller medical clinics. Their position titles are often called Primary Nurse or PA (Physician Assistant). According to the study by Eom [4] out of 114 surgery affiliated training hospitals, 35 hospitals use various position titles for different persons of practice-assisting manpower. 
These reports are usually based on few inconsistent surgery units or surgery rooms, thus data collected from ECC in hospitals do not exist. As expected, with ECC patients increasing and the number of ECC doctors and residents remaining constant, the workload in the ECC is overwhelming, as reported by several studies $[2,5,6]$ conducted among doctors working in the emergency care settings. In order to solve this problem, many countries including the United States have insisted on the utilization of PAs that were properly trained in a given amount of time and education to obtain a national license. Once their requirements are fulfilled, they can perform their duties as a PA under the guidance of a doctor [7].

As indicated in a previous study [8] utilization of PA in the ECC as such can significantly decrease the amount of work for emergency care doctors. Whether this statistical decrease in the workload of ECC doctors is directly related to quality of patient care needs more evidence, but its possibility can be confirmed in the future with larger data.

The cited study above in part focuses on primary care nurses from in-patient and surgery rooms, and thus differs from this study, which focuses on data collected from the emergency care settings. According to our research, all the hospitals included in our study used practice-assisting manpower at minimum of four to maximum of eight persons. This means that by converting to average working hours per weekly, at least one person of practice-assisting manpower working during both day and night was responsible for a significant portion of the workload conducted in the emergency department at each hospital. In addition, their scope of work varied from practice-assisting to procedure-assisting, indicating that their duties in the emergency department are largely distributed over various functions.

Of the procedure-assisting tasks, wound dressing consumed the most time; given that participating hospitals of the research are all high-level facilities of emergency surgery, we can assume that many patients were transferred patients from lower-level hospitals, thus the relative workload required of wound dressing is significantly high. In addition, tasks of checking in patients and inputting test results also showed to consume high number of work hours.

Because these participating hospitals use computerized medical systems for graphs of electrocardiography, the workload for this task along with inputting data into electronic systems require significant number of people and time, as one can infer from our study. Without any practice-assisting manpower, these trivial tasks are assumed to be performed by ECC professionals, and therefore the need of manpower is further increased in the emergency departments. Moreover, based on our data, no time was allocated to seven personal activities of the total 16 observations, indicating that the workload level of practice-assisting manpower is already overwhelming as is.

For example, among procedure-assisting tasks, the blood sampling and intravenous line insertion had the highest total number of work tasks. Although it is often performed with intravenous line insertion, which is considered as first-ranked emergency medical technician's duty, it is not officially specified. In addition, in the case of electrocardiography, it was often conducted by practice-assisting manpower in the majority of the hospitals included in this study. However, the regulation of emergency medical technicians' duties in South Korea does not include any information regarding the matter; rather, an enforcement decree regarding emergency medical technicians considered electrocardiography as a job for clinical laboratory technicians.

As shown, although the duties of emergency medical technicians are regulated, in places where many patients are treated in a short period of time, the duties of doctors and practice-assisting manpower are not clearly separated. Therefore, in cases of possible medical disputes in the future or considering the rights of patients to receiving appropriate medical care, it is necessary to determine appropriate solutions by accurately investigating the matter at national level.

\section{Limitations}

This study was sampled from university hospitals of certain specific regions, thus it contains a few limitations. First, it is difficult to conclude that the observations reflect the work of practice-assisting manpower over the entire day, given the monitoring was conducted only one hour per dayshift and per nightshift. Also, generalization to the national practiceassisting manpower is a limitation, assuming data of this study is from a small sample size of only few university hospitals of certain restricted regions.

Second, although the amount of work varies according to the number of visiting patients of the ECC, this variable was not controlled for in our study. Furthermore, another variable that was not controlled for in the study was the possible difference in gender-determined types of work performed by practiceassisting manpower.

The number of emergency department visiting patients in ECC, the correlations among different types of duties, and particular tasks varying by gender of practice-assisting manpower should all be considered in further studies. There may also be possible statistical bias in that many observations were made at convenient times when the emergency departments are not as busy and in need of less work. Lastly, although we tried to keep the tasks of all observed hospitals constant, there may be variance in how each observer of different hospitals categorized the tasks performed.

\section{Conclusion}

Although the tasks performed by practice-assisting manpower at university hospitals near Daejeon Metropolitan City and Chungcheong Province regions of South Korea can be over the regulated scope of duties, the study showed the significance of these persons over various functions of work in the emergency department. Furthermore, the extent of workload and specific tasks of practice-assisting manpower working in the ECC are in need of extensive discussion. 


\section{Acknowledgements}

This work (NRF-2015R1D1A1A01057890) was supported by NRF.

\section{References}

1. Ahn D. The end of internship training in South Korea. J Korean Med Assoc 2013; 56: 352.

2. Chisholm CD, Weaver CS, Whenmouth L, Giles B. A task analysis of emergency physician activities in academic and community settings. Ann Emerg Med 2011; 58: 117-122.

3. Lee B, Kim H, Lee E. Response plan study on legal issues in utilization of medical support person. Korea Med Assoc Res INST healthcare Policy 2011; 112.

4. Eom YH, Kim GY, Jung SS, Lee KY, Kang S-B, Bae JS. Present condition analysis of physician assistant in Korea. J Korean Med Sci 2012; 27: 981.

5. Kee R, Knott JC, Dreyfus S, Lederman R, Milton S, Joe K. One hundred tasks an hour: An observational study of emergency department consultant activities: One hundred tasks an hour: ED consultant activity. Emerg Med Aust 2012; 24: 294-302.
6. Zhu JN, Weiland TJ, Taylor DM, Dent AW. An observational study of emergency department intern activities. Med J Aust 2008; 188: 514-519.

7. Hooker RS, Klocko DJ, Luke Larkin G. Physician assistants in emergency medicine: the impact of their role: PA ED roles. Acad Emerg Med 2011; 18: 72-77.

8. Brown R. Activities of accident and emergency consultants-a time and motion study. J Accid Emerg Med 2000; 17: 122-125.

\section{*Correspondence to}

Seung Whan Kim

Department of Emergency Medicine

College of Medicine

Chungnam National University Hospital

Daejeon

Republic of Korea 